\title{
AKARI NEAR-INFRARED SPECTROSCOPY OF 3 MICRON PAH AND 4 MICRON PAD FEATURES
}

\author{
T. Onaka ${ }^{1}$, I. Sakon ${ }^{1}$, R. Ohsawa ${ }^{1}$, T. Shimonishi ${ }^{1}$, Y. Okada ${ }^{2}$, \\ M. Tanaka ${ }^{3}$ and H. Kaneda ${ }^{4}$
}

\begin{abstract}
Near-infrared (NIR; $2.5-5 \mu \mathrm{m})$ low-resolution $(\lambda / \Delta \lambda \sim 100)$ spectra were obtained for a number of Galactic and extragalactic objects with the Infrared Camera (IRC) in the AKARI warm mission. These data provide us with the first opportunity to make a systematic study of the $3.3-3.5 \mu \mathrm{m}$ PAH features in a galactic scale as well as within an object. Whereas the $3.3 \mu \mathrm{m}$ band is well resolved in most spectra, the $3.5 \mu \mathrm{m}$ band is not clearly separated from the $3.4 \mu \mathrm{m}$ band in the IRC spectrum. The intensity ratio of the summation of the 3.4 and $3.5 \mu \mathrm{m}$ bands to the $3.3 \mu \mathrm{m}$ band shows a tendency to increase towards the Galactic center, although a large variation in the ratio is also seen in a local scale. A search for deuterated PAH features in the $4 \mu \mathrm{m}$ region is carried out in IRC NIR spectra. Emission lines originating from the ionized gas together with the detector anomaly hamper an accurate search at certain wavelengths, but little convincing evidence has so far been obtained for the presence of significant features in $4.2-4.7 \mu \mathrm{m}$. A conservative upper limit of a few percents is obtained for the integrated intensity ratio of the $4.4-4.7 \mu \mathrm{m}$ possible features to the $3.3-3.5 \mu \mathrm{m}$ PAH features in the spectra so far obtained.
\end{abstract}

\section{Introduction}

The presence of infrared band features (hereafter PAH bands) in the diffuse Galactic radiation, which are attributed to polycyclic aromatic hydrocarbons (PAHs) or PAH-related materials, has been first confirmed for the $3.3 \mu \mathrm{m}$ band by the AROME balloon experiment (Giard et al. 1988) followed by observations with

\footnotetext{
1 Department of Astronomy, Graduate School of Science, University of Tokyo, Tokyo 1130033, Japan; e-mail: onaka@astron.s.u-tokyo.ac.jp

2 I. Physikalisches Institut, Universität zu Köln, 50937 Köln, Germany

3 Center for Computational Sciences, University of Tsukuba, Ibaraki 305-8577, Japan

4 Graduate School of Science, Nagoya University, Aichi 464-8602, Japan
} 
the Infrared Telescope in Space (IRTS; Tanaka et al. 1996). Detection of midinfrared PAH bands in the interstellar medium (ISM) was made simultaneously with IRTS (Onaka et al. 1996) and Infrared Space Observatory (ISO; Mattila et al. 1996). Since then a number of investigations have been carried out for the variations in the MIR PAH bands in Galactic and extragalactic objects including the diffuse Galactic radiation (e.g., Chan et al. 2001; Peeters et al. 2002; Sakon et al. 2004; Rapacioli et al. 2005; Berné et al. 2007; Smith et al. 2007) based on IRTS, ISO and Spitzer observations. However no systematic study has been made for the $3 \mu \mathrm{m}$ PAH band features in a galactic scale except for the studies of the band variation among a small number of objects and within an object by using ground-based telescopes (e.g., Geballe et al. 1989; Joblin et al. 1996; Song et al. 2003) because of a scarcity of sensitive spectrometers in the $3 \mu \mathrm{m}$ region.

$A K A R I$, the Japanese satellite mission dedicated to infrared astronomy (Murakami et al. 2007), used up 180 liter liquid Helium in 2007 August, 550 days after the launch, and completed its cold mission. However, the on-board cryocooler still keeps the telescope and instrument low enough to continue near-infrared (NIR) observations with the Infrared Camera (IRC; Onaka et al. 2007). The IRC has a low-resolution spectroscopic capability (Ohyama et al. 2007) in addition to the imaging, which enables us to carry out sensitive spectroscopic observations in the NIR. In this paper we present the latest results of a study of the $3 \mu \mathrm{m}$ PAH bands based on $A K A R I /$ IRC spectroscopic observations carried out during the $A K A R I$ warm mission (Onaka et al. 2010) together with the results of a search for features of deuterated PAHs in the $4 \mu \mathrm{m}$ region.

\section{IRC spectroscopy and results}

The IRC spectroscopy has the slit and slit-less modes with a choice of the dispersers: prism (NP) and grism (NG) (see Ohyama et al. 2007 for details). For slit-less point source spectroscopy with the grism, a small window is used to avoid overlapping with other sources. A number of various types of objects, including young stellar objects, planetary nebulae, and external galaxies, have so far been observed in this mode in the $A K A R I$ warm mission (e.g., Shimonishi et al. 2010).

The IRC slit spectroscopy has a choice of the slit: medium (Ns) and narrow (Nh). The spectral resolution with Ns is about 100, whereas Nh provides about 150. Both are long slits $(\sim 0$ ' 8$)$ and spatial information can be extracted along the slit. The data used in the present study were taken in the Ns slit spectroscopy. The observations were carried out as part of the $A K A R I$ mission program Interstellar Medium in our Galaxy and nearby galaxies (ISMGN; Kaneda et al. 2009). More than 100 Galactic objects, most of which are HII-PDR complexes, and about 100 positions on the Galactic plane (diffuse Galactic radiation) have been observed in this program, part of which are used in the present investigation.

Figure 1 shows examples of the spectra of the Galactic plane. They clearly detect the 3.3, 3.4, and $3.5 \mu \mathrm{m}$ PAH features together with hydrogen recombination lines originating from the ionized gas (e.g., Br $\alpha$ at $4.05 \mu \mathrm{m}$ and $\operatorname{Br} \beta$ at $2.63 \mu \mathrm{m}$ ). The $3.3 \mu \mathrm{m}$ band is well resolved, but the separation of the 3.4 and $3.5 \mu \mathrm{m}$ bands 

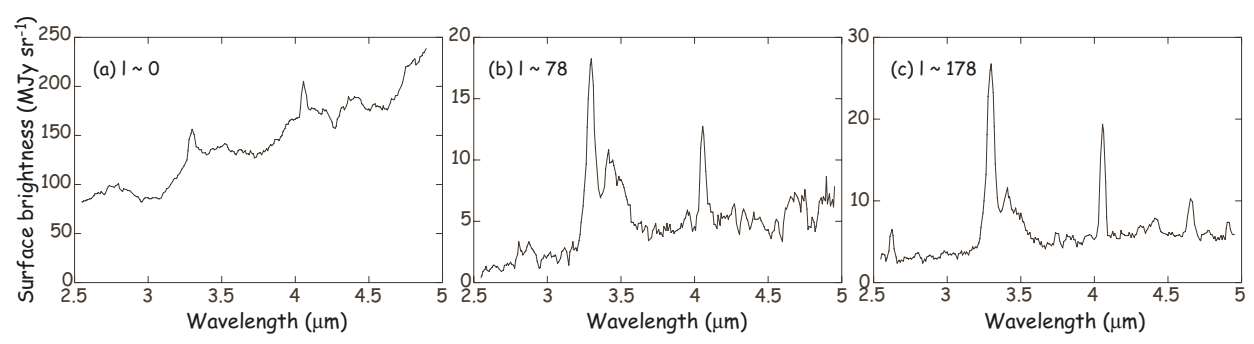

Fig. 1. Examples of AKARI/IRC NIR spectra of the Galactic plane: (a) $l \sim 0^{\circ}$, (b) $l \sim$ $78^{\circ}$, and (c) $l \sim 178^{\circ}$.

is not always clear. The spectra also indicate a significant variation in the PAH features. The spectrum of the Galactic center region (a) shows the absorption features of $\mathrm{H}_{2} \mathrm{O}$ and $\mathrm{CO}_{2}$ ices at 3.0 and $4.3 \mu \mathrm{m}$, respectively, as well as gaseous $\mathrm{CO}$ absorption at $4.6 \mu \mathrm{m}$ and $\mathrm{Br} \alpha$ emission at $4.05 \mu \mathrm{m}$.

To derive the band intensity, we first make a spline fit with the continuum and then fit the PAH features in the $3 \mu \mathrm{m}$ region. A combination of two Lorentzians (for the 3.3 and $3.4 \mu \mathrm{m}$ bands) and one Gaussian (for the $3.5 \mu \mathrm{m}$ band) provides the best fit; however, this does not indicate the nature of the bands because the $3.5 \mu \mathrm{m}$ band consists of more than one components, which are not resolved with the present resolution. Since the separation of the 3.4 and $3.5 \mu \mathrm{m}$ bands is not always clear, only a summation of the two band strengths is discussed in the following.

Figure $2 \mathrm{a}$ shows the intensity ratio of the summation of the 3.4 and $3.5 \mu \mathrm{m}$ bands to the $3.3 \mu \mathrm{m}$ band against that of the 3.4 to $3.3 \mu \mathrm{m}$ band. As described above, the horizontal axis may not be well defined and is used here only for the illustration purpose. The compact sources (mostly HII-PDR complexes) have the ratio in a relatively narrow range $(0.2-0.5)$, while the ratio of the diffuse Galactic radiation shows a large variation. Figure $2 \mathrm{~b}$ plots the intensity ratio in the diffuse radiation against the Galactic longitude. It suggests a global trend that the ratio increases towards the Galactic center region, though the scatter is large. The latter is compatible with the variations seen within an object and among objects in previous studies (e.g., Geballe et al. 1989; Joblin et al. 1996).

\section{Search for deuterated PAH features}

The abundance of deuterium in the ISM is an important parameter in the study of the galaxy evolution since deuterium was formed at the beginning of the Universe and has been gradually destroyed in the stellar interior ("astration"). Its abundance must be strongly linked to the cosmological parameters and the chemical evolution of the Galaxy. Recent far-ultraviolet observations, however, indicate that the interstellar deuterium abundance is significantly lower than model predictions. In addition they suggest several pieces of evidence that deuterium is depleted onto dust grains (Linsky et al. 2006). Draine (2006) proposed that 


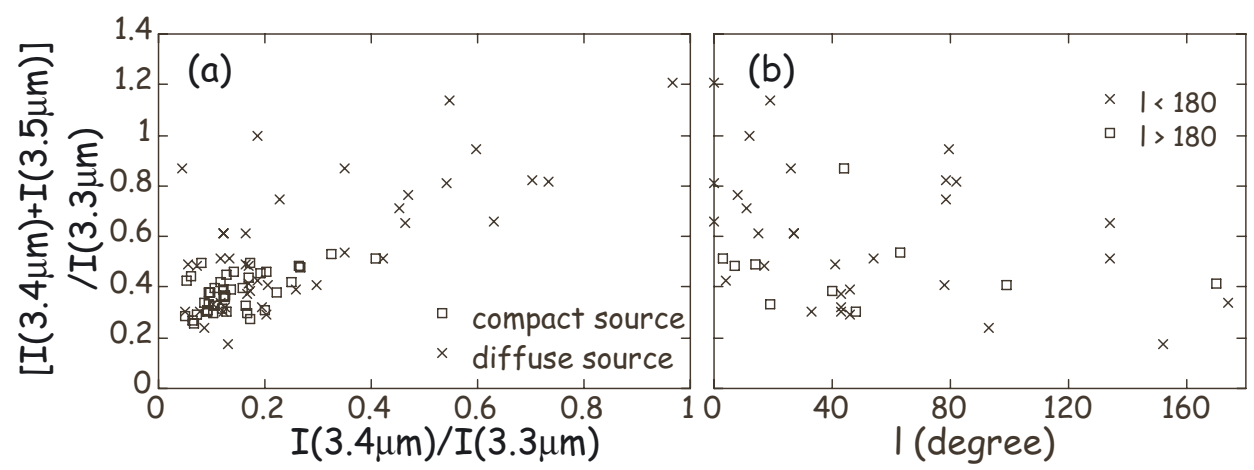

Fig. 2. (a) The intensity ratio of the summation of the 3.4 and $3.5 \mu \mathrm{m}$ bands to the $3.3 \mu \mathrm{m}$ band against that of the 3.4 to $3.3 \mu \mathrm{m}$ band. The open squares indicate "compact" sources, which are mostly HII region-PDR complexes (see text), while the crosses show the diffuse radiation from the Galactic plane. (b) The Galactic longitude dependence of the intensity ratio of the summation of the 3.4 and $3.5 \mu \mathrm{m}$ bands to the $3.3 \mu \mathrm{m}$ band for the diffuse Galactic radiation. The crosses indicate those of $0 \leq l<180^{\circ}$ and the open squares show those of $180 \leq l<360^{\circ}$.

deuterium may be contained in interstellar PAHs since hydrogen in PAHs could be easily replaced by deuterium in low temperature environments. The 3.3 and $3.4 \mu \mathrm{m}$ PAH bands should be shifted to the $4.3-4.7 \mu \mathrm{m}$ region once they are deuterated. If the observed depletion can be attributed solely to deuteration of PAHs, then the deuterium fraction in PAHs can be as large as 0.3. Peeters et al. (2004) reported a possible detection of the 4.4 and $4.65 \mu \mathrm{m}$ features in the Orion bar and M 17 based on ISO/SWS spectra. They derived the integrated intensity ratio of the deuterated PAH features to the 3.3 and $3.4 \mu \mathrm{m}$ PAH features to be $0.17 \pm 0.03$ and $0.36 \pm 0.08$ for the Orion bar and M 17, respectively. However, the detection was marginal even for the best case $(4.4 \sigma)$ and needs to be confirmed by further observations. The IRC spectroscopy offers the best opportunity to search for deuterated PAH features in the $4 \mu \mathrm{m}$, since the predicted spectral range is strongly blocked by the terrestrial atmosphere.

A couple of problems have to be taken into account before making a reliable search for features in the $4 \mu \mathrm{m}$ region in IRC spectra. First, the IRC NIR detector array shows fixed patterns in the direction vertical to the dispersion, which could produce spurious features in the spectrum. Appreciable features are in fact seen occasionally at around $3.8,3.95$, and $4.4 \mu \mathrm{m}$. The last one unfortunately coincides with the wavelength of one of the predicted deuterated PAH features. For the slit-less spectroscopy this does not make a problem since the spurious features can be removed by dithering operations, but it could be a serious problem for the slit spectroscopy. A spectral flat is created for the slit spectroscopy from the spectra of the central part of M 31 by assuming that it is smooth without any appreciable features, and then applied to the observed spectra. 

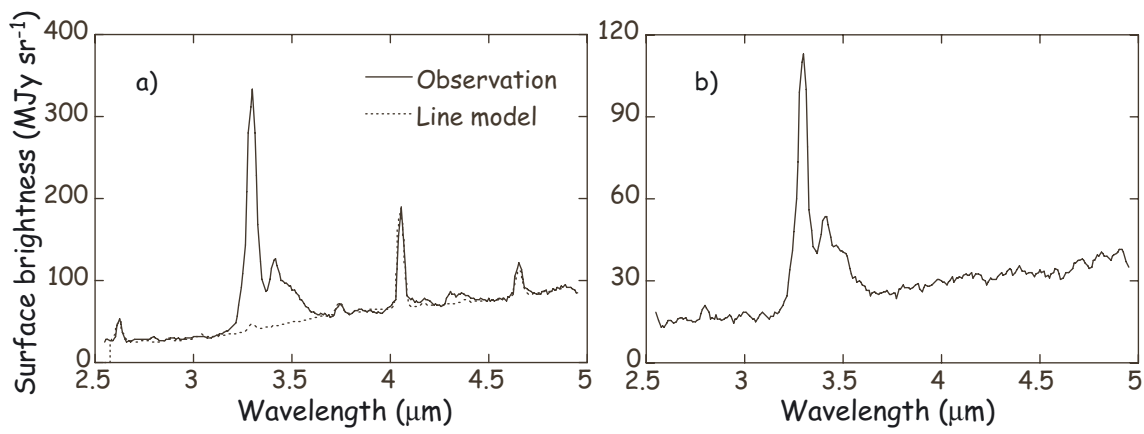

Fig. 3. (a) $A K A R I /$ IRC spectrum of M 17. The solid line shows the observed spectrum corrected for the detector anomaly, whereas the dotted line indicates the spectrum with hydrogen recombination lines that are calculated from $\operatorname{Br} \alpha$ based on the case B conditions (see text). Note that the HeI line at $4.3 \mu \mathrm{m}$ is not included in the calculation. (b) $A K A R I /$ IRC spectrum of a region without recombination lines.

The other issue is the evaluation of the contribution of emission lines from the ionized gas. In particular, $\operatorname{Pf} \beta$ at $4.65 \mu \mathrm{m}$ appears at a wavelength near a predicted feature of deuterated PAHs. Contribution from the ionized gas, if any, must be removed from the spectrum. Assuming the case B conditions with the electron temperature of $7500 \mathrm{~K}$ and the density of $10^{4} \mathrm{~cm}^{-3}$, the line intensity of $\operatorname{Pf} \beta$ is estimated from $\operatorname{Br} \alpha$. The intensities of $\operatorname{Br} \beta$ and $\operatorname{Pf} \gamma$ are also used to estimate possible extinction.

Figure 3a shows an IRC spectrum (solid line) of M 17 together with the spectrum with the predicted line emission (dotted line). The model well accounts for the observed hydrogen emission lines. The observed $4.65 \mu \mathrm{m}$ line seems to be slightly stronger than the predicted $\operatorname{Pf} \beta$. There is also excess emission around $4.3 \mu \mathrm{m}$. Part of the excess can be attributed to the He recombination line at $4.3 \mu \mathrm{m}$, which is not included in the line calculation. Even taking account of possible features remaining around 4.4 and $4.65 \mu \mathrm{m}$, however, the summation of the integrated intensities of these features amounts only $3 \%$ of the summation of the integrated intensities of the $3 \mu \mathrm{m}$ PAH features.

Spectra of the regions that do not have appreciable contribution from the ionized gas have a less uncertainty in the removal of $\operatorname{Pf} \beta$ and could impose a more stringent constraint on the possible feature intensities. Figure $3 \mathrm{~b}$ shows one of the examples, which was obtained towards the Galactic plane of $l \sim 10^{\circ}$. The spectral flat has been applied. It does not show any significant features in the $4 \mu \mathrm{m}$ region, while the $3.3-3.5 \mu \mathrm{m}$ features are clearly seen. A conservative upper limit for the ratio of the integrated intensities is obtained as $2 \%$.

\section{Summary}

The IRC NIR spectroscopy in the $A K A R I$ warm mission provides the first opportunity to make a systematic study of the $3 \mu \mathrm{m}$ PAH features in various objects. 
The intensity ratio of the 3.4 and $3.5 \mu \mathrm{m}$ bands to the $3.3 \mu \mathrm{m}$ band is relatively constant for HII-PDR complexes. The intensity ratio of the diffuse Galactic emission, on the other hand, shows a large variation in a galactic scale, suggesting a tendency that the ratio increases towards the Galactic center region, although the scatter is large. A search for deuterated PAH features in the $4 \mu \mathrm{m}$ region of the IRC spectra of Galactic objects gives a conservative upper limit of $2-3 \%$ for the integrated intensity of the deuterated PAH features relative to the $3.3-3.5 \mu \mathrm{m}$ features. It should be noted that this ratio cannot directly be converted into the abundance ratio because the oscillator strength of isotopic species in the vibration transition could be different. Moreover the difference in the excitation conditions between the 3 and $4 \mu \mathrm{m}$ regions must also be taken into account properly.

This work is based on observations with $A K A R I$, a JAXA project with the participation of ESA. The author thanks all the members of the $A K A R I$ project for their continuous support. This work is supported by a Grant-in-Aid for Scientific Research and a Grant-in-Aid for challenging Exploratory Research from the JSPS.

\section{References}

Berné, O., Joblin, C., Deville, Y., et al., 2007, A\&A, 469, 575

Chan, K.-W., Roellig, T.L., Onaka, T., et al., 2001, ApJ, 231, 183

Draine, B.T., 2006, ASP Conf. Ser., 348, 58

Joblin, C., Tielens, A.G.G.M., Geballe, T.R., \& Wooden, D.H., 1996, ApJ, 458, 610

Geballe, T.R., Tielens, A.G.G.M., Allamandola, L.J., Moorhouse, A., \& Brand, P.W.J.L., 1989, ApJ, 341, 278

Giard, M., Serra, G., Caux, E., Pajot, F., \& Lamarre, J.M., 1988, A\&A, 201, L1

Kaneda, H., Koo, B.-C., Onaka, T., \& Takahashi, H., 2009, Adv. Sp. Res., 44, 1038

Linsky, J., Draine, B.T., Moos, H.W., et al., 2006, ApJ, 647, 1106

Mattila, K., Haikala, L.K., Laureijs, R., et al., 1996, A\&A, 315, L353

Murakami, H., Baba, H., Barthel, P., et al., 2007, PASJ, 59, S309

Ohyama, Y., Onaka, T., Matsuhara, H., et al., 2007, PASJ, 59, S411

Onaka, T., Yamamura, I., Tanabé, T., Roellig, T.L., \& Yuen, L., 1996, PASJ, 48, L59

Onaka, T., Matsuhara, H., Wada, T., et al., 2007, PASJ, 59, S401

Onaka, T., Matsuhara, H., Wada, T., et al., 2010, Proc. SPIE, 7731, 77310M

Peeters, E., Hony, S., Van Kerchhoven, C., et al., 2002, A\&A, 390, 1089

Peeters, E., Allamandola, L.J., Bauschlicher, C.W., Jr., et al., 2004, ApJ, 604, 252

Rapacioli, M., Joblin, C., \& Boissel, P., 2005, A\&A, 429, 193

Sakon, I., Onaka, T., Ishihara, D., et al., 2004, ApJ, 609, 203

Shimonishi, T., Onaka, T., Kato, D., et al., 2010, A\&A, 514, A12

Smith, J.D., Braine, B.T., Dale, D.A., et al., 2007, ApJ, 656, 770

Song, I.-O., Kerr, T.H., McCombie, J., \& Sarre, P.J., 2003, MNRAS, 346, L1

Tanaka, M., Matsumoto, T., Murakami, H., et al., 1996, PASJ, 48, L53 